\title{
Aberrant expression of annexin A10 is closely related to gastric phenotype in serrated pathway to colorectal carcinoma
}

\author{
Jia-Huei Tsai ${ }^{1,2}$, Yu-Lin Lin ${ }^{3,4}$, Yi-Chen Cheng ${ }^{5}$, Chien-Chuan Chen ${ }^{5}$, Liang-In Lin ${ }^{6,7}$, \\ Li-Hui Tseng ${ }^{8}$, Mei-Ling Cheng ${ }^{1}$, Jau-Yu Liau ${ }^{1,2}$ and Yung-Ming Jeng ${ }^{1,2}$
}

${ }^{1}$ Department of Pathology, National Taiwan University Hospital, Taipei, Taiwan; ${ }^{2}$ Graduate Institute of Pathology, College of Medicine, National Taiwan University, Taipei, Taiwan; ${ }^{3}$ Department of Oncology, National Taiwan University Hospital, Taipei, Taiwan; ${ }^{4}$ Graduate Institute of Clinical Medicine, College of Medicine, National Taiwan University, Taipei, Taiwan; ${ }^{5}$ Department of Internal Medicine, National Taiwan University Hospital, Taipei, Taiwan; ${ }^{6}$ Department of Laboratory Medicine, National Taiwan University Hospital, Taipei, Taiwan; ${ }^{7}$ Department of Clinical Laboratory Sciences and Medical Biotechnology, College of Medicine, National Taiwan University, Taipei, Taiwan and ${ }^{8}$ Department of Medical Genetics, National Taiwan University Hospital, Taipei, Taiwan

\begin{abstract}
Annexin A10 (ANXA10) is a member of the ANX family that is normally expressed in gastric mucosa. ANXA10 was recently observed to be upregulated in sessile serrated adenoma, a precursor to microsatellite-unstable colorectal cancer. We investigated the use of ANXA10 in diagnosing colorectal carcinoma. In an immunohistochemical analysis, the intensity and quantity of ANXA10, MUC5AC, MUC6 and CDX2 in 123 colorectal carcinomas were graded. We determined the molecular status of $B R A F$ and KRAS mutations, as well as the microsatellite instability status and the CpG island methylator phenotype in all colorectal carcinomas, and subcategorized into four molecular subgroups according to the molecular derangements. Nuclear ANXA10 staining was present in 36 colorectal carcinomas, exhibiting a strong significant association with the BRAF mutation status $(P<0.0001)$ and positive CpG island methylator phenotype $(P<0.0001)$, and a borderline significant association with high levels of microsatellite instability $(P=0.072)$. The ANXA10-positive colorectal carcinomas were frequently positive for MUC5AC and MUC6, and were associated with absent or reduced CDX2 expression (all $\boldsymbol{P}<\mathbf{0 . 0 0 0 1}$ ). According to a classification and regression tree analysis, ANXA10 is a superior marker for the molecular subtyping of colorectal carcinomas and represents a specific marker for colorectal cancers of the serrated pathway. Our results indicated that ANXA10 expression is implicated in gastric programming in serrated-pathway-associated colorectal carcinoma. ANXA10-positive colorectal carcinoma is highly associated with the molecular features of the serrated neoplasia pathway.

Modern Pathology (2015) 28, 268-278; doi:10.1038/modpathol.2014.96; published online 1 August 2014
\end{abstract}

Colorectal cancer can be divided into several molecular subgroups according to the molecular alterations that occur during multistep carcinogenesis. Most cases of colorectal cancer develop along the adenoma-carcinoma sequence and are characterized by chromosomal instability, harbor $A P C$ and KRAS mutations in $>90 \%$ and approximately $50 \%$ of cases, respectively. ${ }^{1}$ Another molecular pathway

Correspondence: Dr J-Y Liau, MD or Professor Y-M Jeng, MD, PhD, Department of Pathology, National Taiwan University Hospital, 7 Chung-Shun South Road, Taipei 10002, Taiwan.

E-mail: 019188@ntuh.gov.tw or mrna0912@yahoo.com.tw

Received 14 March 2014; revised 20 May 2014; accepted 21 May 2014; published online 1 August 2014 exhibiting high levels of microsatellite instability is caused by genetic inactivation of mismatch repair proteins, either through germline mutation (Lynch syndrome) or epigenetic silencing of the $\mathrm{CpG}$ islands of the promoter (sporadic). ${ }^{1,2}$ A prevailing consensus over the past 2 decades is that sessile serrated adenoma is a precursor lesion of sporadic microsatellite-unstable colorectal carcinoma ${ }^{1-3}$ and, probably, CpG island-methylated microsatellite-stable carcinoma. ${ }^{1,4}$ The tumorigenesis of sessile serrated adenoma is driven by a high rate of $B R A F$ mutation and reinforced by the CpG island methylator phenotype. $^{1-3,5}$ Previous studies have proposed that the aberrant promoter methylation of $\mathrm{CpG}$ islands causes epigenetic silencing of tumor-suppressor 
genes and results in neoplastic progression of sessile serrated adenoma. ${ }^{2,6}$ These findings indicate the possibility that the molecular model of colorectal cancers can be refined based on the microsatellite instability, $\mathrm{CpG}$ island methylator phenotype and $B R A F$ mutation statuses.

A previous study recently identified annexin A10 (ANXA10) as a useful marker of sessile serrated adenoma by conducting gene expression profiling of sessile polyps. ${ }^{7}$ ANXA10 is one of 13 members of the ANX family, ${ }^{8}$ which is a large multigene family of calcium and phospholipid-binding proteins that serve diverse physiologic functions in membrane transport, the calcium signaling pathway, and cell differentiation and proliferation. ${ }^{8-11}$ The ANXs often exhibit aberrant regulation in neoplasia and have garnered considerable interest regarding their association with tumor development and progression. ${ }^{8}$ Some members of the ANX family have crucial roles as oncogenes or tumor suppression genes in various forms of neoplasia and may exhibit contrary expression in various types of cancer. ${ }^{8}$ For example, ANXA1 expression increases in hepatocellular carcinoma and adenocarcinoma of the esophagus, stomach, colon and pancreas, but is downregulated in esophageal squamous cell carcinoma and prostatic adenocarcinoma. ${ }^{8}$ ANXA2 expression increases in adenocarcinoma of the stomach, colon, breast and pancreas, but decreases in prostatic adenocarcinoma. ${ }^{8}$ ANX expression has been correlated with patient survival and tumor stage. 8,12,13 Therefore, human ANXs can exhibit oncogenic and/or tumor-suppressing activities, depending on the cell context. In previous studies, we have shown that ANXA10 is a protein specifically expressed in the gastric mucosa, the Brunner gland of the duodenum and the urothelium. ${ }^{14,15}$ The upregulation of ANXA10 in sessile serrated adenoma may indicate an aberrant gastric phenotype in colonic serrated neoplasia; as evidenced by the high expression of gastric foveolar (MUC5AC) and pyloric mucin (MUC6) in sessile serrated adenoma. ${ }^{16,17}$ Colorectal cancers exhibiting molecular features related to serrated neoplasia pathways are closely associated with MUC5AC and MUC6 expression, ${ }^{18}$ implying that gastric programming participates in the carcinogenesis of a subset of colorectal cancers. This study explored the immunohistochemical expression of ANXA10 in a wide variety of colorectal carcinomas to determine the role of its expression in colorectal cancer.

\section{Materials and methods}

A total of 123 cases were selected from the colorectal cancer cases surgically resected from 2005 to 2013 and had sent for molecular analysis in the Department of Pathology, National Taiwan University Hospital. As the purpose of this study was to compare the expression of ANXA10 in different molecular types of colorectal cancer, we included a study group of 68 cases had at least one of the following molecular features: (1) MSI-H $(n=28)$, (2) $B R A F$ mutation $(n=38)$ and (3) CpG island methylator phenotype $(n=46)$ in this study. For a comparison, a control group of 55 cases of conventional pathwayassociated colorectal cancer diagnosed in the same period was randomly selected for this study. Formalin-fixed paraffin-embedded tissue blocks of primary colorectal carcinoma were retrieved from the archives of the Department of Pathology, National Taiwan University Hospital. The study procedures were conducted according to the regulations of the Research Ethics Committee of National Taiwan University Hospital. A pathologist (J-H Tsai) reviewed the tissue sections and recorded the following pathologic features: Crohn's-like lymphoid reaction (3 or more lymphoid nodular aggregations at the invasive edge of the tumor per $4 \mathrm{X}$ field), tumor-infiltrating lymphocytes (3 or more intraepithelial lymphocytes per 40X field) and dirty necrosis ( $>10 \%$ of the tumor dimensions), ${ }^{19}$ as well as the presence or absence of a mucinous component (any portion), medullary histology or signet ring cell histology. Tumors were graded as well, moderately and poorly differentiated, and designated as right sided if located in the cecum, ascending colon, transverse colon and hepatic flexure and as left sided if located in the descending colon, sigmoid colon, rectosigmoid junction and rectum.

All of the specimens were subjected to molecular analysis and immunohistochemical staining. Molecular classification was performed based on the criteria proposed by Jass et al. ${ }^{20}$ To simplify the statistical correlations of molecular subtypes, we classified carcinomas into the following four molecular subgroups by using a simplified scheme: subgroup 1 consisted of presumed sporadic microsatellite-unstable colorectal carcinomas with microsatellite-unstable/positive CpG island methylator phenotype and/or $B R A F$ mutations; subgroup 2 consisted of serrated-pathway-associated colorectal carcinomas with microsatellite-stable/positive $\mathrm{CpG}$ island methylator phenotype and/or BRAF mutations; subgroup 3 consisted of conventional-pathwayassociated colorectal carcinomas with microsatellitestable and/or KRAS mutations; and subgroup 4 consisted of Lynch-syndrome-like colorectal carcinomas with the microsatellite-unstable/negative CpG island methylator phenotype and without $B R A F$ mutation.

\section{Immunohistochemistry}

Tissue sections (5 $\mu \mathrm{m}$ thick) were dewaxed and rehydrated. Sections of mismatch repair proteins were stained on a Leica Microsystems Bondmax autostainer according to the manufacturer's protocol. Primary antibodies against the following proteins were used: MLH1 (clone ES05, prediluted; Leica 
Biosystems, Newcastle, UK), MSH2 (clone 25D12, prediluted; Leica Biosystems), PMS2 (clone A16-4, 1:100; BD Pharmingen, CA, USA) and MSH6 (clone EPR3945, 1:100; Abcam, Cambridge, MA, USA). Antigen retrieval for other primary antibodies was performed by incubating slides in a $0.01 \mathrm{~m}$ citric acid buffer (pH 6.0) at $100{ }^{\circ} \mathrm{C}$ for $10 \mathrm{~min}$. After blocking the sections with $3 \% \mathrm{H}_{2} \mathrm{O}_{2}$ and $5 \%$ fetal bovine serum, they were incubated with primary antibodies at $4{ }^{\circ} \mathrm{C}$ overnight. The following primary antibodies were used: ANXA10 (1:500, rabbit polyclonal, homemade), MUC5AC (clone CLH2, 1:150; Leica Biosystems), MUC6 (clone CLH5, 1:150; Leica Biosystems) and CDX2 (1:100, Dako Cytomation, Carpinteria, CA, USA). The slides were subsequently incubated with polyclonal goat anti-mouse and anti-rabbit immunoglobulin G secondary antibodies (Dako Cytomation, Glostrup, Denmark). The tissue sections were stained with a diluted 3, 3'-diaminobenzidine tetrachloride solution (Dako Cytomation) and counterstained with hematoxylin. A section of adult gastric mucosa served as a positive control in each immunohistochemical run. Any nuclear immunostaining, with or without cytoplasmic staining, for ANXA10 was considered to be positive and graded semiquantitatively according to the extent of expression (no expression, 0; expression in $<25 \%$ of tumor cells, $1+$; expression in $25 \%-50 \%$ of tumor cells, $2+$; and expression in $>50 \%$ of tumor cells, $3+$ ) and staining intensity (weak, moderate and strong). Positive staining was observed as a nuclear staining pattern in CDX2 and cytoplasmic membranous pattern in MUC5AC and MUC6. CDX2 expression was classified as high (>50\%), reduced $(0-50 \%)$ or absent $(0 \%)$. Negative expression for mismatch repair proteins was defined as complete absence of expression in tumor cells with concurrent positive labeling in non-lesional tissues.

\section{Mutation Analysis}

The nonneoplastic and tumorous areas from $10-\mu \mathrm{m}$ paraffin sections were separately dissected using sterilized razors under a microscope. The nontumorous areas were at least $2 \mathrm{~mm}$ from the tumor. Genomic DNA was extracted using a QIAamp DNA FFPE Tissue Kit (Qiagen, Santa Clarita, CA, USA) according to the manufacturer's protocol. The samples were subjected to a polymerase chain reaction using pairs of primers encompassing exon 15 of $B R A F$ (5'-TCATAATGCTTGCTCTGATAGGA- ${ }^{\prime}{ }^{\prime}$ and $5^{\prime}$-GGC CAAAAATTTAATCAGTGGA-3') and exon 2 of KRAS (5'-GAATGGTCCTGCACCAGTAA- $3^{\prime}$ and $5^{\prime}$-GTGTGACATGTTCTAATATAGTCA-3'). After purification, direct sequencing was performed using an automated ABI 3730 sequencer (Applied Biosystems, Foster City, CA, USA).

The methylation statuses of five marker loci (p16, MINT1, MINT2, MINT31 and MLH1) were evaluated using the Methylight assay. Genomic DNA was treated with sodium bisulfite according to the recommendations of the EZ DNA Methylation Kit protocol (Zymo Research, Orange, CA, USA); the normalization control reaction was based on the methylation-independent measurement of $A l u$ repeats. The methylation levels measured for the five $\mathrm{CpG}$ island methylator phenotype loci in the samples and a constant reference sample were subjected to a quantitative DNA methylation analysis. The percentage of methylated reference was calculated using an equation provided previously, ${ }^{21}$ and loci with percentage of methylated reference values $>10$ were considered to be methylated. CpG island methylator phenotype was designated as positive when 3 or more methylated loci were identified, and negative when $<3$ methylated loci were identified.

The microsatellite instability status was determined using multiplex polymerase chain reaction amplification of five mononucleotide microsatellite loci (BAT-25, BAT-26, NR-21, NR-24 and MONO-27). Amplified polymerase chain reaction products were labeled with fluorescent markers and run on the ABI 3130 capillary electrophoresis instrument (Applied Biosystems). Allelic sizes were analyzed using Genescan analysis software (Applied Biosystems) and differences in the allelic size between normal tissue and the tumors were compared. A tumor was designated as microsatellite unstable when the allelic size varied in two or more microsatellite loci, whereas all other tumors were grouped together as microsatellite stable.

\section{Statistical Analysis}

Data were analyzed using SPSS 19.0 software (IBM Corp., Armonk, NY, USA). Comparisons of categorical variables were performed using the Pearson $\chi^{2}$ method. Continuous variables were analyzed using the Student's $t$-test. A classification and regression tree (CART) analysis was conducted to analyze the value of immunohistochemical expression in molecular classification of colorectal carcinoma. An algorithm was used to define an optimal partition factor in categorizing molecular subtypes of colorectal carcinoma. All statistical results were considered significant when $P<0.05$.

\section{Results}

\section{Molecular Subtypes of Colorectal Carcinoma}

$B R A F$ and KRAS mutations were detected in 38 $(31 \%)$ and $23(19 \%)$ of 123 colorectal cancers in a mutually exclusive manner. A total of 46 cases were positive for $\mathrm{CpG}$ island methylator phenotype. A positive $\mathrm{CpG}$ island methylator phenotype was highly associated with $B R A F$ mutation, but not with the KRAS mutation status $(P<0.0001$ and $P=0.85$, respectively). The status of microsatellite instability 
of all colorectal carcinomas was determined using the polymerase chain reaction of microsatellite loci. Twenty-eight cases of microsatellite-unstable colorectal carcinoma were identified and subjected to mismatch repair protein immunostaining. Figure 1 shows a flowchart of colorectal cancers stratified into four molecular subtypes based on the molecular derangements. Of the 28 microsatellite-unstable cases, 18 were stratified as sporadic microsatelliteunstable colorectal carcinoma (subgroup 1) based on the $B R A F$ mutation status $(n=11)$ and/or positive CpG island methylator phenotype $(n=17)$ status. All colorectal carcinomas in molecular subgroup 1 exhibited concurrent loss of MLH1/PMS2 immunostaining. The other 10 microsatellite-unstable carcinomas were Lynch-syndrome-like colorectal carcinomas (subgroup 4), which exhibited concurrent loss of MLH1/PMS2 $(n=5)$ or MSH2/MSH6 $(n=2)$, or isolated loss of PMS2 $(n=1)$ immunohistochemical expression and had neither $B R A F$ mutation nor CpG island methylator phenotype. One case showed loss of MSH2 but focal and weak MSH6 staining in $<10 \%$ of tumor cells. This case also exhibited positive $\mathrm{CpG}$ island methylator phenotype. Another case showed focal and weak MSH2/MSH6 staining in $<10 \%$ of tumor cells. Both cases exhibited microsatellite instability by polymerase chain reaction of microsatellite loci, and they are classified into subgroup 4. No isolated loss of MSH6 staining was observed in any cases. We classified 40 microsatellite-stable colorectal carcinomas into subgroup 2; these carcinomas exhibited $B R A F$ mutation $(n=27)$ and/or positive CpG island methylator phenotype $(n=28)$. The molecular alterations of molecular subgroups 1 and 2 were considered closely related to the serrated neoplastic pathways. $^{20}$ Another 55 colorectal cancers were included in molecular subgroup 3, in which we observed KRAS mutation in 14 (26\%) cases. A comparison of the histological features of the molecular subgroups is summarized in Table 1.

\section{ANXA10 Staining is Correlated with Specific Clinicopathologic Features}

Of the 123 cases, 36 (29\%) exhibited immunoreactivity to ANXA10 (Figure 2). The intratumoral distribution of immunostaining was heterogeneous, ranging from $<25 \%$ in $67 \%$ of the cases, $25-50 \%$ in $19 \%$ of the cases and $>50 \%$ in $14 \%$ of the cases. The staining intensity varied from weak (in $50 \%$ of the cases) to moderate (19\%) to strong (31\%) expression. ANXA10 expression was closely associated with certain clinicopathologic features of colorectal cancer (Table 2) and exhibited a highly significant correlation with the right-sided tumor location $(P=0.001)$. There was a strong association of ANXA10 expression with moderate to poor differentiation of tumor grading, Crohn's-like lymphoid reaction and a lack of dirty necrosis (all $P<0.0001$ ). All five colorectal carcinomas with signet ring cell histology expressed ANXA10. No correlations with the pathologic features of mucinous differentiation, medullary histology and tumor-infiltrating lymphocytes were observed $(P=0.226,0.967$ and 0.135 , respectively).

\section{The Role of ANXA10 Expression in the Gastric Programming of Serrated Neoplasms to Colorectal Carcinoma}

Strong immunoreactivity associations were observed between ANXA10 and the expression of the other two gastric mucins: MUC5AC and MUC6 (both

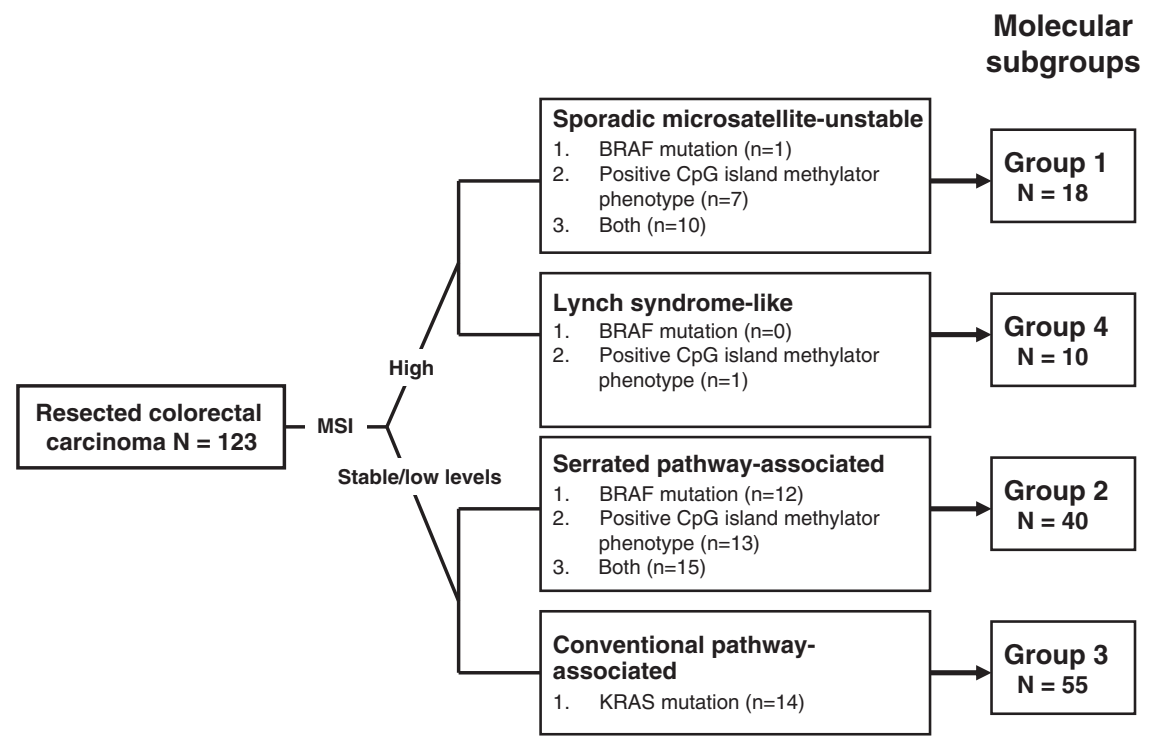

Figure 1 A flowchart detailing the 123 colorectal carcinomas stratified into four molecular subgroups according to the molecular derangements. 
Table 1 Pathologic features related to the four molecular subgroups of colorectal carcinomas

\begin{tabular}{|c|c|c|c|c|c|}
\hline & \multicolumn{4}{|c|}{ Molecular subgroups } & \multirow[b]{2}{*}{$\mathrm{P}$-value } \\
\hline & $\begin{array}{c}1 \\
\mathrm{~N}=18\end{array}$ & $\begin{array}{c}2 \\
\mathrm{~N}=40\end{array}$ & $\begin{array}{c}3 \\
\mathrm{~N}=55\end{array}$ & $\begin{array}{c}4 \\
\mathrm{~N}=10\end{array}$ & \\
\hline \multicolumn{5}{|l|}{ Location (n (\%)) } & \multirow[t]{3}{*}{$<0.0001$} \\
\hline Right & $17(94 \%)$ & $20(50 \%)$ & $13(24 \%)$ & $8(80 \%)$ & \\
\hline Left & $1(6 \%)$ & $20(50 \%)$ & $42(76 \%)$ & $2(20 \%)$ & \\
\hline \multicolumn{5}{|l|}{ Adjacent precursor lesion } & \multirow[t]{4}{*}{0.007} \\
\hline Tubular adenoma/tubulovillous adenoma & 2 & 3 & 10 & 3 & \\
\hline Sessile serrated adenoma & 1 & 0 & 0 & 0 & \\
\hline Traditional serrated adenoma & 0 & 4 & 0 & 0 & \\
\hline \multicolumn{5}{|l|}{ Tumor differentiation (n (\%)) } & \multirow[t]{4}{*}{$<0.0001$} \\
\hline Well & $1(6 \%)$ & $9(23 \%)$ & $34(62 \%)$ & $2(20 \%)$ & \\
\hline Moderate & $2(11 \%)$ & $13(32 \%)$ & $15(27 \%)$ & $4(40 \%)$ & \\
\hline Poor & $15(83 \%)$ & $18(45 \%)$ & $6(11 \%)$ & $4(40 \%)$ & \\
\hline \multicolumn{6}{|l|}{ Tumor histology (n (\%)) } \\
\hline Mucinous differentiation & $11(61 \%)$ & $6(15 \%)$ & $12(22 \%)$ & $6(60 \%)$ & $<0.0001$ \\
\hline Medullary histology & $6(33 \%)$ & $0(0 \%)$ & $0(0 \%)$ & $1(10 \%)$ & $<0.0001$ \\
\hline Signet ring histology & $4(22 \%)$ & $0(0 \%)$ & $0(0 \%)$ & $1(10 \%)$ & $<0.0001$ \\
\hline \multicolumn{6}{|l|}{ Pathologic features of invasive carcinoma (n (\%)) } \\
\hline Crohn's-like reaction & $18(100 \%)$ & $27(68 \%)$ & $19(35 \%)$ & $8(80 \%)$ & $<0.0001$ \\
\hline Tumor-infiltrating lymphocytes & $13(72 \%)$ & $7(18 \%)$ & $5(9 \%)$ & $8(80 \%)$ & $<0.0001$ \\
\hline Dirty necrosis & $2(11 \%)$ & $20(50 \%)$ & $43(78 \%)$ & $5(50 \%)$ & $<0.0001$ \\
\hline
\end{tabular}

$P<0.0001$ ) (Figure 3). Of the 36 ANXA10-positive colorectal carcinomas, $33(92 \%)$ expressed either MUC5AC or MUC6. Twenty-one cases (58\%) of ANXA10-positive colorectal carcinomas exhibited the concurrent expression of MUC5AC and MUC6. In addition, immunohistochemical expression of ANXA10, MUC5AC and MUC6 was highly correlated with absent or reduced expression of CDX2 (all $P<0.0001)$. Of 37 colorectal cancers exhibited absent or reduced CDX2 expression, 34 (92\%) stained for either ANXA10 $(n=22)$, MUC5AC $(n=30)$ or MUC6 $(n=24)$.

\section{Correlation of Immunostaining with Molecular Features and Subtypes of Colorectal Carcinoma}

Table 3 is a summary of the correlation between the molecular features of colorectal carcinoma and immunohistochemical profiles. Positive immunoexpression of ANXA10, MUC5AC and MUC6 was highly associated with molecular derangements, namely the positive $\mathrm{CpG}$ island methylator phenotype and $B R A F$ mutation. An inverse association of the positive $\mathrm{CpG}$ island methylator phenotype and $B R A F$ mutation with high CDX2 expression was observed. KRAS-mutated colorectal carcinomas were more often negative for ANXA10 and MUC6 and positive for CDX2 than were the KRAS wild type $(P=0.016,0.022$ and 0.048 , respectively). We observed a strong association between the microsatellite-unstable status and MUC6 and MUC5AC staining, as well as absent or reduced CDX2 expression $(P<0.0001,0.001$ and 0.002 , respectively). A borderline significant association of ANXA10 expression was found with microsatellite-unstable status $(P=0.072)$. There was a strong significant relationship between the immunohistochemical expression for ANXA10, MUC5AC, MUC6 and high CDX2 expression and the molecular subgroups. To determine the best immunohistochemical marker for the molecular subtyping of colorectal carcinoma, we conducted a CART analysis using immunohistochemical expressive results of ANXA10, MUC5AC, MUC6 and CDX2. The splitting algorithm is shown in Figure 4. The molecular phenotypes of colorectal carcinoma were adequately explained by the immunostaining for ANXA10, MUC5AC and MUC6 with a prediction rate of $67 \%$. CDX2 provided no significant contribution in the model. The optimal partition factor was ANXA10 expression, followed by MUC6 and MUC5AC expression. The first split node at ANXA10 expression successfully separated subgroups 1 and 2 (both presumably serrated-pathway-associated colorectal carcinomas) from subgroups 3 and 4 colorectal carcinoma (conventional-pathway and Lynchsyndrome-like colorectal carcinomas, respectively) with a positive predictive value of $95 \%$ and a negative predictive value of $74 \%$. Other ANXA10negative cases in subgroups 1 and 2 were separated from subgroups 3 and 4 at the second and third nodes according to MUC6 and MUC5AC expression, respectively. 
a
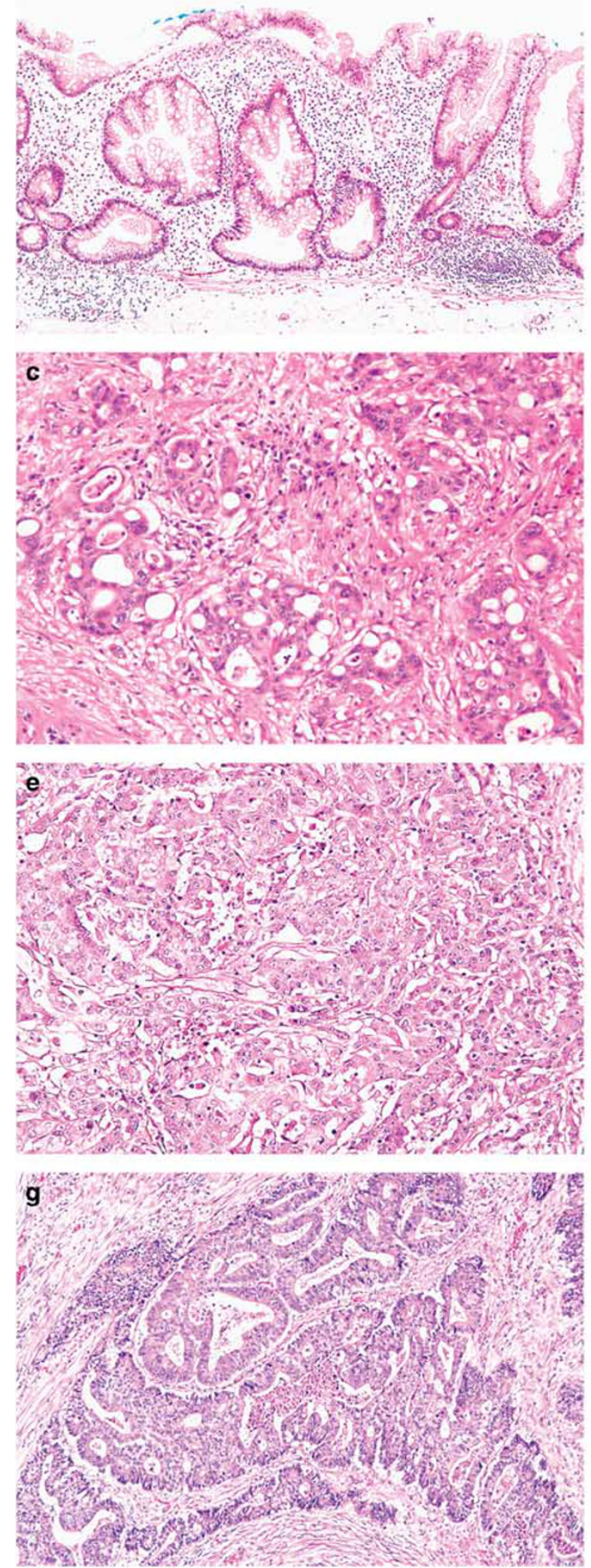

b
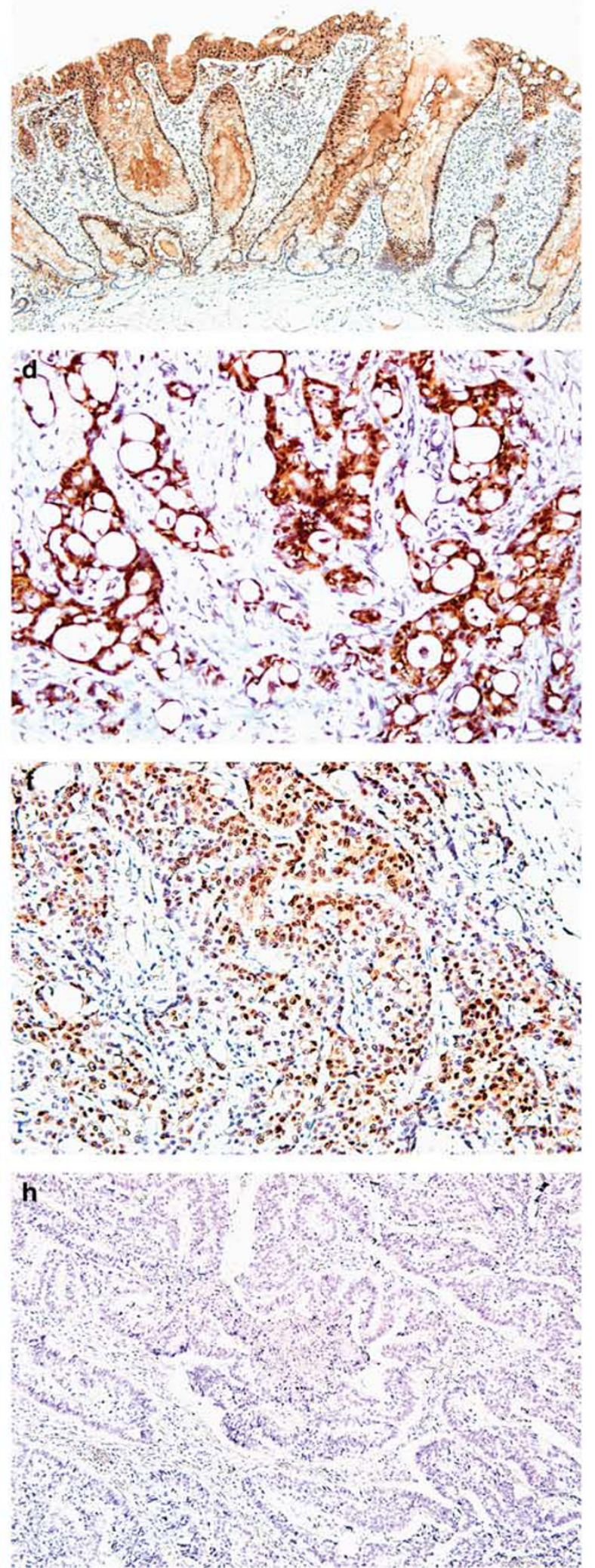

Figure 2 Immunohistochemical expression for annexin A10 (ANXA10). Panels (a, b) showed a typical example of sessile serrated adenoma strongly staining for ANXA10. Panels (c, d) were a BRAF-mutated colorectal carcinoma showing strong expression for ANXA10. Panels (e, f) showed ANXA10 expression in a microsatellite-unstable colorectal carcinoma. Panels (g, h) showed a conventional-pathway-associated colorectal carcinoma, which was negative for ANXA10. 
Table 2 Clinicopathologic features related to ANXA10 expression

\begin{tabular}{|c|c|c|c|c|}
\hline & ANXA10 positive $\mathrm{N}=36$ & ANXA10 negative $\mathrm{N}=87$ & Odds ratio & $\mathrm{P}$-value \\
\hline Location (n (\%)) & & & 3.72 & 0.001 \\
\hline Right & $25(69 \%)$ & $33(38 \%)$ & & \\
\hline Left & $11(31 \%)$ & $54(62 \%)$ & & \\
\hline Tumor differentiation (n (\%)) & & & - & $<0.0001$ \\
\hline Well & $4(11 \%)$ & $42(48 \%)$ & & \\
\hline Moderate & $8(22 \%)$ & $26(30 \%)$ & & \\
\hline Poor & $24(67 \%)$ & $19(22 \%)$ & & \\
\hline \multicolumn{5}{|l|}{ Tumor histology (n (\%)) } \\
\hline Mucinous differentiation & $13(36 \%)$ & $22(25 \%)$ & 1.67 & 0.226 \\
\hline Medullary histology & $2(6 \%)$ & $5(6 \%)$ & 0.97 & 0.967 \\
\hline Signet ring histology & $5(14 \%)$ & $0(0 \%)$ & - & $<0.0001$ \\
\hline \multicolumn{5}{|c|}{ Pathologic features of invasive carcinoma (n (\%)) } \\
\hline Crohn's-like reaction & $30(83 \%)$ & $42(48 \%)$ & 5.36 & $<0.0001$ \\
\hline Tumor-infiltrating lymphocytes & $13(36 \%)$ & $20(23 \%)$ & 1.89 & 0.135 \\
\hline Dirty necrosis & $10(28 \%)$ & $60(69 \%)$ & 0.17 & $<0.0001$ \\
\hline \multicolumn{5}{|l|}{ MUC5AC expression } \\
\hline Positive & $33(92 \%)$ & $34(39 \%)$ & 17.15 & $<0.0001$ \\
\hline Negative & $3(8 \%)$ & $53(61 \%)$ & & \\
\hline MUC6 expression & & & 4.69 & $<0.0001$ \\
\hline Positive & $21(58 \%)$ & $20(23 \%)$ & & \\
\hline Negative & $15(42 \%)$ & $67(77 \%)$ & & \\
\hline CDX2 expression & & & 7.54 & $<0.0001$ \\
\hline$<50 \%$ & $22(61 \%)$ & $15(17 \%)$ & & \\
\hline$\geqq 50 \%$ & $14(39 \%)$ & $72(83 \%)$ & & \\
\hline Molecular subgroup of carcinoma & & & - & $<0.0001$ \\
\hline Group $1(n=18)$ & $10(28 \%)$ & $8(9 \%)$ & & \\
\hline Group $2(n=40)$ & $24(67 \%)$ & $16(18 \%)$ & & \\
\hline Group $3(n=55)$ & $0(0 \%)$ & $55(63 \%)$ & & \\
\hline Group $4(n=10)$ & $2(6 \%)$ & $8(9 \%)$ & & \\
\hline
\end{tabular}

Abbreviation: ANXA10, annexin A10.

\section{Discussion}

Colorectal cancer is one of the most prevalent cancers worldwide. The incidence is higher in developed countries than in developing countries, and epidemiological studies have implicated dietary habits, lifestyle and environmental elements as crucial factors in the etiology of colorectal cancer. ${ }^{1}$ Although the molecular mechanisms underlying these etiological factors are poorly characterized, complex interactions among dietary patterns, the host lifestyle and the intestinal microbiota contribute in affecting the biologic microenvironment in the large intestine. ${ }^{1,22,23}$ Studies have reported that distinct tumor milieu in the proximal and distal segments of the large intestine associated with discrete mutation patterns. ${ }^{24}$ In one study, colonic adenomas from various anatomic locations of the colon exhibited distinct DNA methylation clusters. ${ }^{25}$ Studies have shown that the de novo regulation of gastric-type mucin (MUC5AC and MUC6) is predominantly observed in proximally located colorectal cancers, ${ }^{18}$ and higher MUC6 expression was observed in right-sided serrated polyps than left- sided based on the extent and staining intensity. ${ }^{16,17}$ We observed that, in addition to MUC5AC and MUC6, ANXA10 immunoreactivity was highly associated with the right sidedness of the large intestine. As ANXA10 is not expressed in the normal colon mucosa, ${ }^{7,14}$ the upregulation of ANXA10 indicates that the right and left segments of the large intestine have unique tumorigenetic backgrounds.

Consistent with previous observations, ${ }^{18}$ we observed that the expression of MUC5AC and MUC6 apomucins was strongly associated with molecular features of the serrated neoplastic pathway, such as BRAF mutation, the $\mathrm{CpG}$ island methylator phenotype and microsatellite instability. Moreover, we noted an association between these molecular alterations and ANXA10 positivity. MUC5AC and MUC6 are gel-forming mucins secreted by gastric foveolar cells and pyloric glands, respectively. ${ }^{26}$ MUC6 has a cytoprotective role in forming a mucous barrier against hostile gastric acid. ${ }^{27}$ As ANXA10 was characterized to have a role in gastric gland development and maintenance, ${ }^{14}$ it was not surprised that ANXA10 expression was highly associated with MUC5AC and MUC6 positivity. 

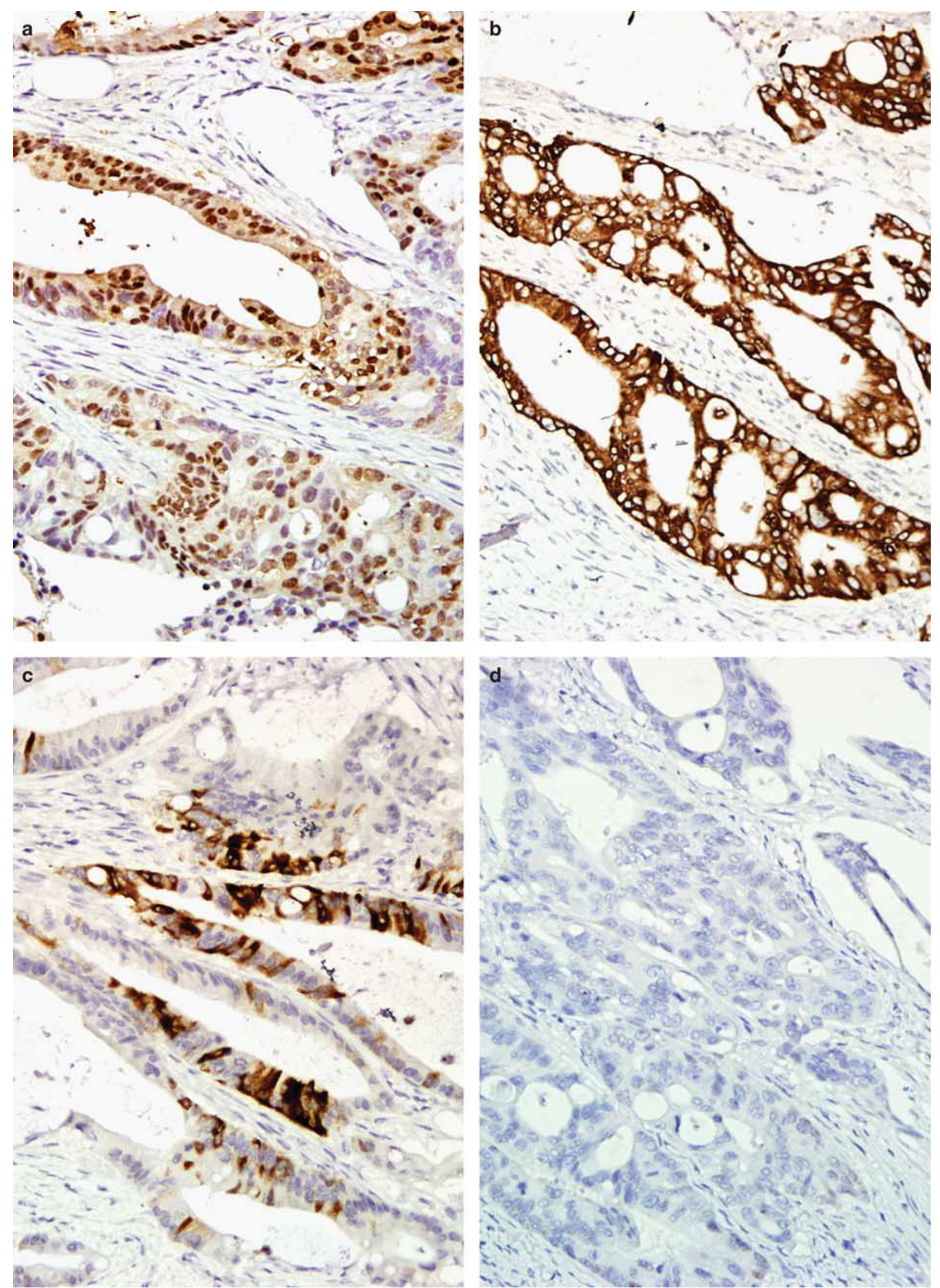

Figure 3 An annexin A10-positive (a) colorectal carcinoma also stained for MUC5AC (b), MUC6 (c) but was negative for CDX2 (d). 
Table 3 Comparison of positive immunohistochemical staining correlated to molecular alterations

\begin{tabular}{|c|c|c|c|c|c|c|c|c|}
\hline & \multicolumn{2}{|c|}{ ANXA10 positive } & \multicolumn{2}{|c|}{ MUC5AC positive } & \multicolumn{2}{|c|}{ MUC6 positive } & \multicolumn{2}{|c|}{ CDX2 high expression } \\
\hline & $\mathrm{N}=36$ & $\mathrm{P}$-value & $\mathrm{N}=67$ & $\mathrm{P}$-value & $\mathrm{N}=41$ & $\mathrm{P}$-value & $\mathrm{N}=86$ & $\mathrm{P}$-value \\
\hline BRAF mutation & & $<0.0001$ & & $<0.0001$ & & $<0.0001$ & & $<0.0001$ \\
\hline Present $(n=38)$ & $27(75 \%)$ & & $32(48 \%)$ & & $22(54 \%)$ & & $16(19 \%)$ & \\
\hline Absent $(n=85)$ & $9(25 \%)$ & & $35(52 \%)$ & & $19(46 \%)$ & & $70(81 \%)$ & \\
\hline KRAS mutation & & 0.016 & & 0.24 & & 0.022 & & 0.048 \\
\hline Present $(n=23)$ & $2(6 \%)$ & & $10(15 \%)$ & & $3(7 \%)$ & & $20(23 \%)$ & \\
\hline Absent $(n=100)$ & $34(94 \%)$ & & $57(85 \%)$ & & $38(93 \%)$ & & $66(77 \%)$ & \\
\hline CpG island methylator phenotype & & $<0.0001$ & & 0.001 & & $<0.0001$ & & $<0.0001$ \\
\hline Positive $(n=46)$ & $26(72 \%)$ & & $34(51 \%)$ & & $28(68 \%)$ & & $20(23 \%)$ & \\
\hline Negative $(n=77)$ & $10(28 \%)$ & & $33(49 \%)$ & & $13(32 \%)$ & & $66(77 \%)$ & \\
\hline Microsatellite status & & 0.072 & & 0.001 & & $<0.0001$ & & 0.002 \\
\hline Unstable $(n=28)$ & $12(33 \%)$ & & $23(34 \%)$ & & $20(49 \%)$ & & $13(15 \%)$ & \\
\hline Stable $(n=95)$ & $24(67 \%)$ & & $44(66 \%)$ & & $21(51 \%)$ & & $73(85 \%)$ & \\
\hline Molecular subgroup of carcinoma & & $<0.0001$ & & $<0.0001$ & & $<0.0001$ & & $<0.0001$ \\
\hline Group $1(n=18)$ & $10(28 \%)$ & & $17(25 \%)$ & & $16(39 \%)$ & & $6(7 \%)$ & \\
\hline Group $2(n=40)$ & $24(67 \%)$ & & $27(40 \%)$ & & $16(39 \%)$ & & $23(27 \%)$ & \\
\hline Group $3(n=55)$ & $0(0 \%)$ & & $17(25 \%)$ & & $5(12 \%)$ & & $50(58 \%)$ & \\
\hline Group $4(n=10)$ & $2(6 \%)$ & & $6(9 \%)$ & & $4(10 \%)$ & & $7(8 \%)$ & \\
\hline
\end{tabular}

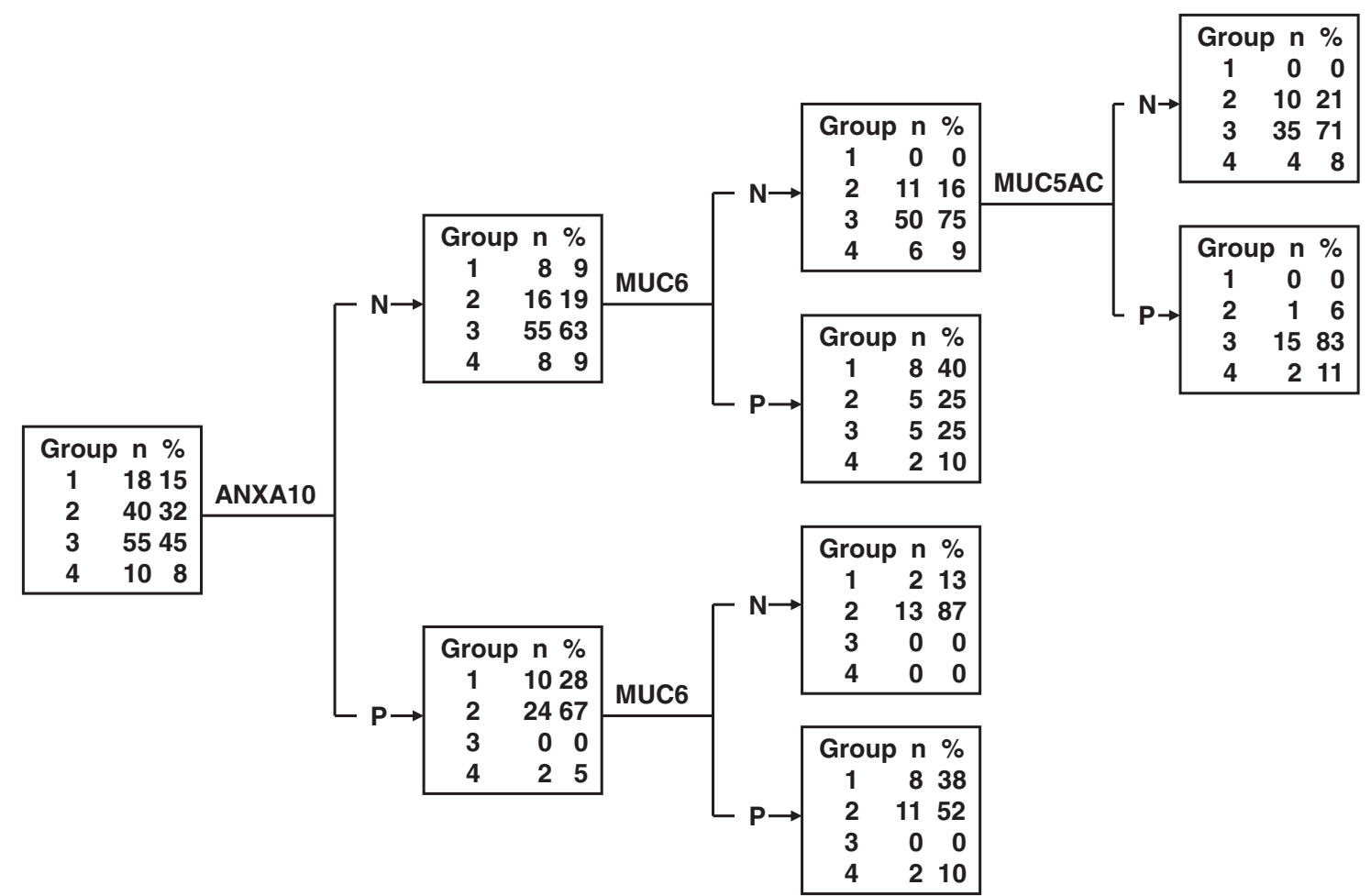

Figure 4 Classification and regression tree (CART) model of immunohistochemical staining of annexin A10 (ANXA10), MUC5AC, MUC6 and high expression of CDX2. The model targets for molecular subgrouping of colorectal carcinomas with a statistical significance. The results indicated that ANXA10 was the most important partition factor. First split was created by the presence of ANXA10 expression and successfully separated the majority of subgroups 3 and 4 colorectal carcinomas from subgroups 1 and 2 . The second and third nodes were split at MUC6 and MUC5AC expression, respectively, which made further contribution to molecular subclassification of the colorectal carcinomas in ANXA10-negative branch. CDX2 provided no significant contribution in the model.

Yao et $a l^{28}$ first described gastric differentiation in serrated adenoma in 1999. Subsequent studies have confirmed the simultaneous activation of gastric mucins in serrated neoplasms and associated invasive carcinomas. ${ }^{16-18}$ Aberrant gastric metaplasia was evident based on an inverse association 
of ANXA10 expression with CDX2 reactivity, which is a key factor in intestinal differentiation. ${ }^{18}$ As both serrated precursors and the associated carcinomas highly expressed gastric-phenotype-associated proteins (MUC5AC, MUC6 and ANXA10), ${ }^{7,16-18}$ a core signaling pathway toward gastric programming may indicate that a distinct phenotype is involved in the tumorigenesis of serrated neoplasia. In our study, ANXA10 was consistently negative in conventionalpathway-associated colorectal carcinomas. By contrast, nonspecific expression levels of MUC5AC and MUC6 were observed relatively commonly in subgroups 3 and 4 of colorectal carcinomas (mean percentile, $20 \%$ and $50 \%$, respectively). Consequently, ANXA10 is a superior marker to MUC5AC and MUC6 in predicting molecular features related to the serrated neoplastic pathway. ANXA10 is located on chromosome $4 \mathrm{q} 33$ and is distinct from other human ANXs, with rare tissue expression and a loss of type II calcium-binding sites in three of four conserved ANX repeats. ${ }^{29}$ The ANX repeats are an evolutional conserved carboxy-terminal core domain with a highly packed $\alpha$-helical module of 4 or 8 repeats of 70 amino-acid residuals and serve as calcium-binding sites. ${ }^{9,30}$ In contrast to the carboxyterminal protein core, the amino-terminal domain of each ANX member varies in length and sequence. The amino-terminus sequences often harbor 16 or fewer residues and regulate conformation of the molecule, providing a binding site for target protein ligands. The unique structure of the ANXs facilitates a flexible interaction with associated molecules, and ANXs function as membrane scaffold proteins to implement diverse physiologic functions in a reversible manner. ${ }^{9,30}$

The ANX proteins are also involved in regulating the epidermal growth factor receptor (EGFR) signaling transduction pathway. ${ }^{31}$ EGFR is a crucial cellsurface protein that is activated when it is bound to specific extracellular stimulatory signals. EGFR activation promotes a complex series of intracellular responses, thus mediating various cellular activities. To execute signaling transduction, the ANXs have a critical role in modulating reciprocal regulation and synergistic interactions in the response cascades. ${ }^{31}$ The mitogen-activated protein kinase (MAPK) pathway is a branch of the essential downstream cascades of EGFR signaling and contributes to the cellular proliferation, neoplastic transformation and cell differentiation of tumors. ${ }^{32}$ The ANX members ANXA1, ANXA2, ANXA6 and ANXA8 were determined to downregulate the EGFR/MAPK cascade. ${ }^{31}$ The mechanisms of each member are heterogeneous, but largely mediate in a $\mathrm{Ca}^{2+}$-dependent manner. ${ }^{31}$ By contrast, a previous study reported that ANXA10, with a decrease of $\mathrm{Ca}^{2+}$-binding sites, was associated with MAPK pathway activation and the promotion of cell proliferation. ${ }^{33}$ This finding is consistent with our finding that ANXA10 expression is closely associated with the $B R A F$ mutation status, which causes constitutive activation of the MAPK pathway. Therefore, a decrease of calcium-binding sites in ANXA10 may impart a unique functional consequence with a biological linkage with MAPK pathway activity in the serrated carcinogenesis of colorectal cancers.

A recent study suggested that the coexpression of mucin core proteins (MUC2, MUC5AC and MUC6) in colorectal carcinomas is stimulated by the activation of the EGFR/BRAF/MAPK signaling pathway. ${ }^{18} \mathrm{~A}$ decrease of CDX2 expression may also be induced by downstream effectors of RAF/MAPK activity. ${ }^{34}$ In our study, reduced CDX2 was highly associated with microsatellite-unstable colorectal carcinomas (subgroup 1) and carcinomas with $B R A F$ mutation or $\mathrm{CpG}$ methylator phenotype statuses (subgroup 2). This observation supported the view that decreased expression of CDX2 could be caused by regulatory mechanisms in addition to the epigenetic silencing of the CDX2 promoter in colorectal carcinoma. ${ }^{18,35}$ These findings imply that gastric programming is an integral process in serrated neoplasia through the EGFR/BRAF/MAPK pathway and subsequently by $\mathrm{CpG}$ island methylator phenotype. Although the exact mechanisms of the interactive processes between ANXA10 and the mucin core proteins remain unknown, an increasing recognition of the gastric phenotype in serrated neoplasia provides a pathogenetic picture of colorectal cancer.

In summary, this study investigated the immunohistochemical expression of ANXA10 in a large number of colorectal cancers. Aberrant expression of ANXA10 was associated with right-sided colorectal carcinoma, the $B R A F$ mutation and the positive $\mathrm{CpG}$ island methylator phenotype status. Immunostaining of ANXA10 often indicated positivity for other gastric mucins (MUC5AC and MUC6) and absent or decreased CDX2 expression. This study proposes a gastric phenotype in a subset of colorectal carcinomas and extends our knowledge of the pathogenesis of the serrated carcinogenetic pathway.

\section{Acknowledgments}

We thank the staff of the Second Core Laboratory, Department of Medical Research, National Taiwan University Hospital, for providing technical support. This work was supported by grant NTUH. 103-N2449 from the National Taiwan University Hospital.

\section{Disclosure/conflict of interest}

The authors declare no conflict of interest.

\section{References}

1 Snover DC, Ahnen DJ, Burt RW, et al. Serrated polyps of the colon and rectum and serrated polyposis, In: Bosman FT, Carneiro F, Hruban RH, Theise ND. (eds). WHO Classification of Tumours of the Digestive 
System, 4th edn. International Agency for Research on Cancer: Lyon, France; 2010, pp 160-165.

2 Bettington M, Walker N, Clouston A, et al. The serrated pathway to colorectal carcinoma: current concepts and challenges. Histopathology 2013;62:367-386.

3 Rex DK, Ahnen DJ, Baron JA, et al. Serrated lesions of the colorectum: review and recommendations from an expert panel. Am J Gastroenterol 2012;107:1315-1329.

4 Jass JR. Serrated adenoma of the colorectum and the DNA-methylator phenotype. Nat Clin Pract Oncol 2005;2:398-405.

5 Kambara T, Simms LA, Whitehall VL, et al. BRAF mutation is associated with DNA methylation in serrated polyps and cancers of the colorectum. Gut 2004;53:1137-1144.

6 Jass JR, Whitehall VL, Young J, et al. Emerging concepts in colorectal neoplasia. Gastroenterology 2002;123:862-876.

7 Gonzalo DH, Lai KK, Shadrach B, et al. Gene expression profiling of serrated polyps identifies annexin A10 as a marker of a sessile serrated adenoma/polyp. J Pathol 2013;230:420-429.

8 Mussunoor S, Murray GI. The role of annexins in tumour development and progression. J Pathol 2008;216:131-140.

9 Rescher U, Gerke V. Annexins-unique membrane binding proteins with diverse functions. J Cell Sci 2004;117:2631-2639.

10 Gerke V, Creutz CE, Moss SE. Annexins: linking Ca2 + signalling to membrane dynamics. Nat Rev Mol Cell Biol 2005;6:449-461.

11 Schlaepfer DD, Haigler HT. Expression of annexins as a function of cellular growth state. J Cell Biol 1990;111: 229-238.

12 Stein T, Price KN, Morris JS, et al. Annexin A8 is upregulated during mouse mammary gland involution and predicts poor survival in breast cancer. Clin Cancer Res 2005;11:6872-6879.

13 Emoto K, Sawada H, Yamada Y, et al. Annexin II overexpression is correlated with poor prognosis in human gastric carcinoma. Anticancer Res 2001;21: 1339-1345.

$14 \mathrm{Lu} \mathrm{SH}$, Chen YL, Shun CT, et al. Expression and prognostic significance of gastric-specific annexin A10 in diffuse- and intestinal-type gastric carcinoma. J Gastroenterol Hepatol 2011;26:90-97.

$15 \mathrm{Lu} \mathrm{SH}$, Yuan RH, Chen YL, et al. Annexin A10 is an immunohistochemical marker for adenocarcinoma of the upper gastrointestinal tract and pancreatobiliary system. Histolpathol 2013;63:640-648.

16 Bartley AN, Thompson PA, Buckmeier JA, et al. Expression of gastric pyloric mucin, MUC6, in colorectal serrated polyps. Mod Pathol 2010;23:169-176.

17 Gibson JA, Hahn HP, Shahsafaei A, et al. MUC expression in hyperplastic and serrated colonic polyps: lack of specificity of MUC6. Am J Surg Pathol 2011;35:742-749.

18 Walsh MD, Clendenning M, Williamson E, et al. Expression of MUC2, MUC5AC, MUC5B, and MUC6 mucins in colorectal cancers and their association with the CpG island methylator phenotype. Mod Pathol 2013;26:1642-1656.

19 Bellizzi AM, Frankel WL. Colorectal cancer due to deficiency in DNA mismatch repair function: a review. Adv Anat Pathol 2009;16:405-417.

20 Jass JR. Classification of colorectal cancer based on correlation of clinical, morphological and molecular features. Histopathology 2007;50:113-130.

21 Weisenberger DJ, Siegmund KD, Campan M, et al. CpG island methylator phenotype underlies sporadic microsatellite instability and is tightly associated with BRAF mutation in colorectal cancer. Nat Genet 2006;38:787-793.

22 Chen HM, Yu YN, Wang JL, et al. Decreased dietary fiber intake and structural alteration of gut microbiota in patients with advanced colorectal adenoma. Am J Clin Nutr 2013;97:1044-1052.

23 Fuchs CS, Giovannucci EL, Colditz GA, et al. Dietary fiber and the risk of colorectal cancer and adenoma in women. N Engl J Med 1999;340:169-176.

24 Rosty C, Young JP, Walsh MD, et al. Colorectal carcinomas with KRAS mutation are associated with distinctive morphological and molecular features. Mod Pathol 2013;26:825-834.

25 Koestler DC, Li J, Baron JA, et al. Distinct patterns of DNA methylation in conventional adenomas involving the right and left colon. Mod Pathol 2014;27:145-155.

26 Nordman H, Davies JR, Lindell G, et al. Gastric MUC5AC and MUC6 are large oligomeric mucins that differ in size, glycosylation and tissue distribution. Biochem J 2002;364:191-200.

27 Bartman AE, Buisine MP, Aubert JP, et al. The MUC6 secretory mucin gene is expressed in a wide variety of epithelial tissues. J Pathol 1998;186:398-405.

28 Yao T, Kouzuki T, Kajiwara M, et al. 'Serrated' adenoma of the colorectum, with reference to its gastric differentiation and its malignant potential. J Pathol 1999;187:511-517.

29 Morgan RO, Jenkins NA, Gilbert DJ, et al. Novel human and mouse annexin A10 are linked to the genome duplications during early chordate evolution. Genomics 1999;60:40-49.

30 Gerke V, Moss SE. Annexins: from structure to function. Physiol Rev 2002;82:331-371.

31 Grewal T, Enrich C. Annexins-modulators of EGF receptor signalling and trafficking. Cell Signal 2009;21: 847-858.

32 Barbacid M. ras genes. Annu Rev Biochem 1987;56: 779-827.

33 Shimizu T, Kasamatsu A, Yamamoto A, et al. Annexin A10 in human oral cancer: biomarker for tumoral growth via G1/S transition by targeting MAPK signaling pathways. PloS One 2012;7:e45510.

34 Krueger F, Madeja Z, Hemberger M, et al. Downregulation of $\mathrm{Cdx} 2$ in colorectal carcinoma cells by the Raf-MEK-ERK 1/2 pathway. Cell Signal 2009;21: 1846-1856.

35 Kawai H, Tomii K, Toyooka S, et al. Promoter methylation downregulates CDX2 expression in colorectal carcinomas. Oncol Rep 2005;13:547-551. 